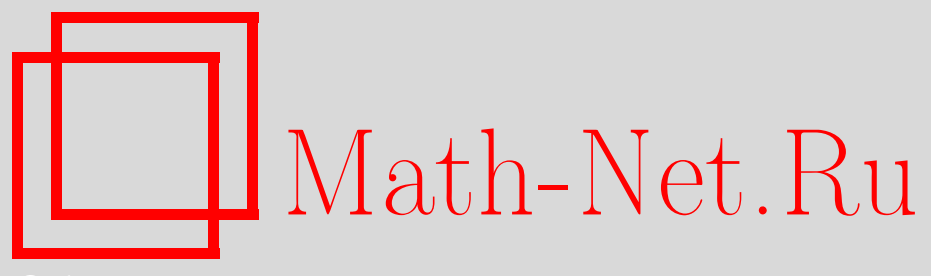

А. Б. Жижченко, А. Д. Изаак, Информационная система Math-Net.Ru. Современное состояние и перспективы развития. Импакт-факторы российских математических журналов, УМН, 2009, том 64, выпуск 4, 195-204

DOI: https://doi.org/10.4213/rm9312

Использование Общероссийского математического портала Math-Net.Ru подразумевает, что вы прочитали и согласны с пользовательским соглашением http://www . mathnet.ru/rus/agreement

Параметры загрузки:

IP : 54.237 .59 .107

26 апреля 2023 г., 13:13:04

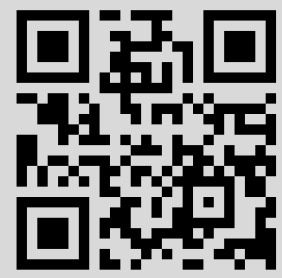




\title{
Информационная система Math-Net.Ru. Современное состояние и перспективы развития. Импакт-факторы российских математических журналов
}

\author{
А. Б. Жижченко, А. Д. Изаак
}

В настоящей заметке мы коротко рассказываем о том, какие изменения произошли в информационной системе Math-Net.Ru (http://www .mathnet.ru) за время, прошедшее с последней публикации (см. [1]), и описываем наши планы на ближайшую перспективу.

Прежде всего, отметим, что существенно возросло (утроилось!) количество участвующих в проекте изданий. Наряду с журналами по чистой математике появилось несколько журналов по прикладной математике. Появился и первый физический журнал - "Успехи физических наук". Бесспорным украшением портала стали семь серий энциклопедического издания ВИНИТИ "Итоги науки и техники".

Открыт раздел "Видеотека", в котором собрано уже более 300 видеозаписей докладов и лекций, прочитанных как российскими, так и зарубежными математиками на различных конференциях, школах и семинарах.

Количество посетителей портала неуклонно растет и в настоящее время составляет более трех с половиной тысяч в день по данным аналитической службы компании Google (Google Analytics, http://www.google.com/analytics). При этом около 60\% посетителей - российские пользователи, $40 \%$ - зарубежные $(20 \%$ - из "ближнего", $20 \%$ - из "дальнего" зарубежья).

Количественные показатели современного состояния Math-Net.Ru приведены в табл. 1.

ТАБлицА 1. Современное состояние портала Math-Net.Ru (на 1.08.2009)

\begin{tabular}{|l|r|}
\hline Журналов: & 51 \\
\hline Публикаций: & 65879 \\
\hline Взаимных ссылок: & 82392 \\
\hline Цитированных статей: & 24226 \\
\hline Записей в БДЦЛ: & 497781 \\
\hline Персоналий: & 26984 \\
\hline Организаций: & 2477 \\
\hline Конференций: & 53 \\
\hline Докладов и лекций: & 312 \\
\hline
\end{tabular}

Появился ряд новых сервисов, главные из которых связаны со списками цитированной литературы.

Практически с самого начала работы над порталом Math-Net.Ru мы стали обрабатывать списки цитированной литературы, снабжая их ссылками на несколько

(C) А. Б. ЖИЖченко, А. Д. ИЗААК, 2009 
баз данных в Интернете (см. [1; п. 2.2.2]). Если публикация - элемент списка - присутствует в базе данных портала Math-Net.Ru, в одной из реферативных баз данных (MathSciNet, http://www.ams.org/mathscinet; Zentralblatt MATH, http://www.zentralblatt-math.org/zmath; ADS NASA, http://adsabs.harvard.edu) или имеет DOI (http://www.crossref.org), она сопровождается ссылками на соответствующие сайты с информацией об этой публикации.

Благодаря этому появились обратные ссылки на публикацию, т.е. списки статей, представленных на портале, которые цитируют данную публикацию. Это дало возможность не только прослеживать историю рассматриваемых в публикации вопросов (благодаря списку иитируемой литературы), но и понять, какое развитие они получили в дальнейшем (благодаря списку цитирующих публикаций).

Однако обратные ссылки появлялись только в том случае, если соответствующая цитирующая статья была опубликована в одном из журналов, представленных на портале. Этого было явно недостаточно - ведь таким образом исключались и еще не участвующие в проекте российские журналы, и, тем более, многочисленные зарубежные журналы (и не только математические!).

Некоторое время тому назад мы начали собирать ссылки на "матнетовские" публикации из всевозможных периодических и продолжающихся изданий, как российских, так и зарубежных. При этом мы используем все доступные источники информации, представленные в Интернете. Работа по сбору таких ссылок только началась, но уже сейчас мы имеем неплохие результаты.

Составление списков цитирующих публикаций - основная цель, ради которой мы занимаемся поиском обратных ссылок. Однако попутно появляется возможность получения различного рода статистической информации - количество цитирований каждого журнала, отдельных статей, авторов, появились списки наиболее цитируемых статей и авторов.

Наибольший интерес у посетителей портала вызвали импакт-факторы по версии Math-Net.Ru.

Мы не будем подробно останавливаться на истории возникновения импакт-факторов. Не будем также углубляться в проблему отношения к подобного рода числовым оценкам научной периодики и, в целом, научной деятельности. Подробный анализ см., например, в [2]. Обсуждение проблем, связанных с импакт-факторами, можно найти в [3]-[7].

Классические "двухлетние" импакт-факторы журналов (будем обозначать их IF ISI) рассчитываются Институтом научной информации корпорации Томсон Рейтер (Information Science Institute (ISI), Thomson Reuters, http://apps.isiknowledge.com). Импакт-фактор журнала за год $N$ - это количество ссылок в году $N$ на статьи журнала, опубликованные в нем в течение двух предыдущих лет $N-2$ и $N-1$, деленное на количество статей, опубликованных в журнале за эти два года $N-2$ и $N-1$.

Импакт-фактор по версии Math-Net.Ru (для краткости будем в дальнейшем писать ИФМ) рассчитывается по этой же схеме и учитывает ссылки из всевозможных периодических и продолжающихся (серийных) изданий на научные статьи журнала. Под "научными" мы понимаем все публикации, кроме статей биобиблиографического характера (юбилеи, некрологи), статей с информацией о конференциях, анонсов различных мероприятий, рецензий, "писем в редакцию" и т. п. Мы не стараемся зафиксировать значение импакт-фактора журнала за определенный год раз и навсегда: по мере уточнения списка цитирующих статей оно может меняться, как правило, в бо́льшую сторону. 
Каковы были причины, по которым мы начали вести подсчет собственного ИФМ, при том, что его формула совпадает с классической? Их несколько. Во-первых, хотелось понять, за счет каких статей (как цитирующих, так и цитируемых) формируются импакт-факторы журналов. Насколько нам известно, ISI не раскрывает эти данные. Во-вторых, лишь для немногих российских журналов вычисляется классический импакт-фактор, что, по нашему мнению, не справедливо (см. табл. 3 ниже). И, в-третьих, судя по всему, далеко не все ссылки из вполне достойных журналов попадают в числитель IF ISI.

При всей кажущейся простоте определения импакт-фактора, при его подсчете начинает возникать целый клубок проблем. Перечислим некоторые из них.

1. Все ведущие российские математические журналы переводятся на английский язык. Англоязычные версии статей, как правило, публикуются в журналах с другим названием, другим издателем, с тем или иным временным лагом. Естественно, при подсчете импакт-фактора должны учитываться ссылки как на русскоязычную, так и на англоязычную версию статьи. Если обе версии журнала выходят почти одновременно или с разницей в два-три месяца, то проблем нет. А если английская версия выходит только через год после русской (например, журнал "Алгебра и анализ")? ИФМ берет за основу русскоязычную версию журнала. Например, ИФМ-2007 журнала "Алгебра и анализ" учитывает, в частности, ссылки на статьи, опубликованные в журнале "St. Petersburg Mathematical Journal" в 2006 и 2007 гг. - именно в них опубликованы переводы статей журнала "Алгебра и анализ" 2005 и 2006 гг.

2. Как определить год $N$ публикации статьи, претендующей попасть в числитель импакт-фактора? Казалось бы, ответ очевидный - это год, который присутствует в выходных данных статьи, именно он и берется при вычислении ИФМ. Однако, судя по всему, ISI считает годом публикации статьи тот момент, когда соответствующий номер журнала приходит к подписчикам. Так, например, ссылки из статей многих декабрьских номеров журналов за 2007 год попадают в числители IF ISI за 2008 год. Всё это вносит дополнительную путаницу в подсчеты IF ISI.

3. Многие зарубежные издательства на своих Интернет-сайтах стали размещать предварительные версии статей, которые со временем попадают в один из текущих номеров журнала. Например, издательство Шпрингер публикует такие статьи на сайте SpringerLink под рубрикой "Online First". При этом год публикации статьи "online" может отличаться от года окончательной публикации. Какой год считать годом публикации? ИФМ считает таким годом год окончательной публикации, хотя со временем, возможно, придется изменить это соглашение.

4. В ISI иногда путают российские журналы с похожими названиями. Нам известен журнал, IF ISI которого завышен из-за учета в числителе ссылок на другие журналы с похожим названием, IF ISI которых не вычисляется.

5. Часто в списках цитируемой литературы можно встретить ссылку на еще не опубликованную в момент написания статьи работу. В базе данных Math-Net.Ru мы стараемся уточнить соответствующую ссылку, чтобы, в частности, учитывать ее при подсчете ИФМ.

6. Нередко авторы статей допускают ошибки при подготовке списков цитируемой литературы, а редакторы не всегда их обнаруживают. В связи с этим при обработке списков литературы мы применяем ряд механизмов, позволяющих находить цитируемые с ошибками статьи в базе данных Math-Net.Ru. K сожалению, для уверенности в правильности полученных результатов поиска полностью исключить здесь ручную работу не удается. Все вышесказанное относится также и к ошибкам набора, в первую очередь для списков цитируемой литературы к статьям, опубликованным в “доэлектронный" период. 
7. В переводной версии статьи список цитируемой литературы может быть изменен. Учитывать ли ссылки, которых не было в оригинальной версии статьи? ИФМ их учитывает.

8. В переводной версии журнала добавлена новая статья (такое, например, в последнее время практикует журнал "Математические заметки"). При подсчете ИФМ мы не учитываем ни саму статью (знаменатель), ни ссылки на нее (числитель).

9. Препринты, опубликованные на сайте arXiv.org, при подсчете ИФМ (в числителе) не учитываются. А что делать, если в статье есть ссылка на arXiv.org, а на самом деле соответствующий препринт уже опубликован в журнале? Нам кажется, что такие ссылки должны учитываться при подсчете импакт-факторов.

Приведем теперь собственно импакт-факторы российских математических журналов и некоторую другую статистическую информацию, доступную на портале Math-Net.Ru. Заметим, что мы не касаемся споров об импакт-факторах, не составляем рейтинги, не делаем никаких выводов. Мы лишь пытаемся раскрыть происхождение самих значений импакт-факторов и, тем самым, даем пищу для размышлений, возможность для анализа на основе полученных данных.

На портале по состоянию на 1 августа 2009 года собраны архивы 51 издания (30 академических - 13 журналов РАН и 17 журналов институтов РАН; 20 университетских изданий; 1 журнал АН Республики Молдова). 36 из них продолжают выходить в настоящее время. ИФМ за 2003-2008 гг. вычислен для тридцати (за исключением "молодых" журналов, а также журналов, недавно включенных в систему Math-Net.Ru, для которых подсчет еще не закончен).

Обработанными (структурированными) списками цитируемой литературы снабжено около половины всех публикаций, содержащихся в базе данных портала, в том числе все статьи 2003-2009 гг. (В "Успехах математических наук" списки литературы обработаны для всех научных статей журнала за период 1967-2009 гг.) Эти списки содержат почти полмиллиона ссылок.

Обратных ссылок в настоящий момент около 80000 . Пока большинство из них "внутриматнетовские" ссылки (т.е. и цитируемая, и цитирующая статьи содержатся в базе данных портала Math-Net.Ru).

\begin{tabular}{|c|c|}
\hline aa - & Алгебра и анализ \\
\hline al - & Алгебра и логика \\
\hline $\mathrm{dm}-$ & Дискретная математика \\
\hline $\mathrm{da}-$ & Дискретный анализ и исследование операций \\
\hline znsl - & Записки научных семинаров ПОМИ \\
\hline ivm - & Известия высших учебных заведений. Математика \\
\hline $\mathrm{izv}-$ & Известия Российской академии наук. Серия математическая \\
\hline $\mathrm{mz}-$ & Математические заметки \\
\hline $\mathrm{mt}-$ & Математические труды \\
\hline $\mathrm{msb}-$ & Математический сборник \\
\hline $\mathrm{mm}-$ & Математическое моделирование \\
\hline $\mathrm{mmj}-$ & Moscow Mathematical Journal \\
\hline ppi - & Проблемы передачи информации \\
\hline sjim - & Сибирский журнал индустриальной математики \\
\hline smj - & Сибирский математический журнал \\
\hline $\operatorname{tmf}-$ & Теоретическая и математическая физика \\
\hline $\operatorname{tm}-$ & Труды Математического института имени В. А. Стеклова \\
\hline umn - & Успехи математических наук \\
\hline fpm - & Фундаментальная и прикладная математика \\
\hline faa - & Функциональный анализ и его приложения \\
\hline
\end{tabular}


ТАБЛицА 2. ИФМ 2003-2008 гг. ряда журналов по состоянию на 1.08.2009

\begin{tabular}{|c|c|c|c|c|c|c|c|c|c|c|}
\hline Год & aa & al & $\mathrm{dm}$ & $\mathrm{da}$ & znsl & ivm & izv & $\mathrm{mz}$ & $\mathrm{mt}$ & $\mathrm{msb}$ \\
\hline \hline 2008 & 0.340 & 0.493 & 0.212 & 0.224 & 0.179 & 0.095 & 0.310 & 0.283 & 0.171 & 0.506 \\
\hline 2007 & 0.533 & 0.589 & 0.149 & 0.206 & 0.292 & 0.064 & 0.400 & 0.244 & 0.219 & 0.399 \\
\hline 2006 & 0.582 & 0.453 & 0.188 & 0.116 & 0.192 & 0.091 & 0.515 & 0.219 & 0.143 & 0.404 \\
\hline 2005 & 0.398 & 0.416 & 0.106 & 0.132 & 0.146 & 0.076 & 0.495 & 0.185 & 0.250 & 0.414 \\
\hline 2004 & 0.489 & 0.408 & 0.058 & 0.138 & 0.261 & 0.084 & 0.564 & 0.179 & 0.094 & 0.494 \\
\hline 2003 & 0.519 & 0.250 & 0.188 & 0.310 & 0.230 & 0.059 & 0.490 & 0.194 & 0.139 & 0.488 \\
\hline
\end{tabular}

\begin{tabular}{|c|c|c|c|c|c|c|c|c|c|c|}
\hline Год & $\mathrm{mm}$ & $\mathrm{mmj}$ & $\mathrm{ppi}$ & $\mathrm{sjim}$ & $\mathrm{smj}$ & $\mathrm{tmf}$ & $\mathrm{tm}$ & $\mathrm{umn}$ & $\mathrm{fpm}$ & faa \\
\hline \hline 2008 & 0.123 & 0.444 & 0.354 & 0.181 & 0.528 & 0.735 & 0.363 & 0.488 & 0.207 & 0.513 \\
\hline 2007 & 0.129 & 0.488 & 0.470 & 0.132 & 0.269 & 0.601 & 0.294 & 0.382 & 0.080 & 0.473 \\
\hline 2006 & 0.137 & 0.716 & 0.400 & 0.117 & 0.254 & 0.708 & 0.193 & 0.441 & 0.129 & 0.429 \\
\hline 2005 & 0.044 & 0.691 & 0.231 & 0.198 & 0.256 & 0.585 & 0.200 & 0.333 & 0.114 & 0.550 \\
\hline 2004 & 0.119 & 0.620 & 0.344 & 0.243 & 0.180 & 0.586 & 0.144 & 0.405 & 0.070 & 0.484 \\
\hline 2003 & 0.084 & 0.508 & 0.169 & 0.184 & 0.234 & 0.217 & 0.188 & 0.485 & 0.060 & 0.394 \\
\hline
\end{tabular}

ТАБлИЦА 3. Сравнение ИФМ и IF ISI 2008 г.

\begin{tabular}{|c|c|c|c|c|c|c|c|c|c|c|}
\hline & aa & al & $\mathrm{dm}$ & $\mathrm{da}$ & znsl & ivm & izv & $\mathrm{mz}$ & $\mathrm{mt}$ & $\mathrm{msb}$ \\
\hline \hline ИФМ & 0.340 & 0.493 & 0.212 & 0.224 & 0.179 & 0.095 & 0.310 & 0.283 & 0.171 & 0.506 \\
\hline IF ISI & - & - & - & - & - & - & 0.494 & 0.270 & - & 0.415 \\
\hline
\end{tabular}

\begin{tabular}{|c|c|c|c|c|c|c|c|c|c|c|}
\hline & $\mathrm{mm}$ & $\mathrm{mmj}$ & $\mathrm{ppi}$ & $\mathrm{sjim}$ & $\mathrm{smj}$ & $\mathrm{tmf}$ & $\mathrm{tm}$ & $\mathrm{umn}$ & $\mathrm{fpm}$ & faa \\
\hline \hline ИФМ & 0.123 & 0.444 & 0.354 & 0.181 & 0.528 & 0.735 & 0.363 & 0.488 & 0.207 & 0.513 \\
\hline IF ISI & - & - & - & - & 0.445 & 0.721 & - & 0.430 & - & 0.449 \\
\hline
\end{tabular}

ТАБЛицА 4. Сравнение ИФМ и IF ISI 2007 г.

\begin{tabular}{|c|c|c|c|c|c|c|c|c|c|c|}
\hline & aa & al & $\mathrm{dm}$ & $\mathrm{da}$ & $\mathrm{znsl}$ & $\mathrm{ivm}$ & $\mathrm{izv}$ & $\mathrm{mz}$ & $\mathrm{mt}$ & $\mathrm{msb}$ \\
\hline \hline ИФМ & 0.533 & 0.589 & 0.149 & 0.206 & 0.292 & 0.064 & 0.400 & 0.244 & 0.219 & 0.399 \\
\hline IF ISI & - & - & - & - & - & - & 0.453 & 0.251 & - & 0.359 \\
\hline
\end{tabular}

\begin{tabular}{|c|c|c|c|c|c|c|c|c|c|c|}
\hline & $\mathrm{mm}$ & $\mathrm{mmj}$ & $\mathrm{ppi}$ & $\mathrm{sjim}$ & $\mathrm{smj}$ & $\mathrm{tmf}$ & $\mathrm{tm}$ & $\mathrm{umn}$ & $\mathrm{fpm}$ & faa \\
\hline \hline ИФМ & 0.129 & 0.488 & 0.470 & 0.132 & 0.269 & 0.601 & 0.294 & 0.382 & 0.080 & 0.473 \\
\hline IF ISI & - & - & - & - & 0.208 & 0.622 & - & 0.309 & - & 0.367 \\
\hline
\end{tabular}


ТАБлица 5. Наиболее цитируемые статьи, представленные на Math-Net.Ru

\begin{tabular}{|c|c|c|}
\hline 1. & $\begin{array}{l}\text { Динамические системы с упругими отражениями. } \\
\text { Эргодические свойства рассеивающих бильярдов } \\
\text { Я. Г. Синай } \\
\text { УМН, 1970, 25:2(152), 141-192 }\end{array}$ & 251 \\
\hline 2. & $\begin{array}{l}\text { Характеристические показатели Ляпунова и гладкая эргодическая теория } \\
\text { Я. Б. Песин } \\
\text { УМН, 1977, 32:4(196), 55-112 }\end{array}$ & 191 \\
\hline 3. & $\begin{array}{l}\text { Квазилинейные уравнения первого порядка } \\
\text { со многими независимыми переменными } \\
\text { С. Н. Кружков } \\
\text { Матем. сб., } 1970, \mathbf{8 1}(\mathbf{1 2 3}): 2,228-255\end{array}$ & 190 \\
\hline 4. & $\begin{array}{l}\text { Нелинейные уравнения типа Кортевега-де Фриза, конечнозонные } \\
\text { линейные операторы и абелевы многообразия } \\
\text { Б. А. Дубровин, В. Б. Матвеев, С. П. Новиков } \\
\text { УМН, 1976, 31:1(187), 55-136 }\end{array}$ & 185 \\
\hline 5. & $\begin{array}{l}\text { Пылевая плазма } \\
\text { В. Е. Фортов, А. Г. Храпак, С. А. Храпак, В. И. Молотков, О. Ф. Петров } \\
\text { УФН, 2004, 174:5, 495-544 }\end{array}$ & 177 \\
\hline 6. & $\begin{array}{l}\text { Малые знаменатели и проблемы устойчивости движения в классической } \\
\text { и небесной механике } \\
\text { В. И. Арнольд } \\
\text { УМН, 1963, 18:6(114), 91-192 }\end{array}$ & 168 \\
\hline 7. & $\begin{array}{l}\text { Гиббсовские меры в эргодической теории } \\
\text { Я. Г. Синай } \\
\text { УМН, 1972, 27:4(166), 21-64 }\end{array}$ & 157 \\
\hline 8. & $\begin{array}{l}\text { Доказательство теоремы А.Н. Колмогорова о сохранении условно- } \\
\text { периодических движений при малом изменении функции Гамильтона } \\
\text { В. И. Арнольд } \\
\text { УМН, } 1963, \mathbf{1 8 : 5}(113), 13-40\end{array}$ & 152 \\
\hline 9. & $\begin{array}{l}\text { Тэта-функции и нелинейные уравнения } \\
\text { Б. А. Дубровин } \\
\text { УМН, 1981, 36:2(218), 11-80 }\end{array}$ & 129 \\
\hline 10. & $\begin{array}{l}\text { Гидродинамика слабо деформированных солитонных решеток. } \\
\text { Дифференциальная геометрия и гамильтонова теория } \\
\text { Б. А. Дубровин, С. П. Новиков } \\
\text { УМН, } 1989, \mathbf{4 4 : 6 ( 2 7 0 ) , 2 9 - 9 8}\end{array}$ & 119 \\
\hline
\end{tabular}

ТАБлицА 6. Наиболее цитируемые авторы портала Math-Net.Ru

\begin{tabular}{|r|l|r|}
\hline 1. & В. И. Арнольд & 1322 \\
\hline 2. & С. П. Новиков & 1071 \\
\hline 3. & Я. Г. Синай & 1030 \\
\hline 4. & И. М. Гельфанд & 832 \\
\hline 5. & В. П. Маслов & 781 \\
\hline 6. & И. М. Кричевер & 633 \\
\hline 7. & О. А. Олейник & 632 \\
\hline 8. & Б. А. Дубровин & 596 \\
\hline 9. & М. И. Вишик & 533 \\
\hline 10. & Л. Д. Фаддеев & 519 \\
\hline
\end{tabular}


При всей неоднозначности понятия цитируемости, согласитесь, списки получаются неслучайными.

Для полноты представления статистической информации, собранной на портале Math-Net.Ru, приведем списки наиболее популярных среди посетителей портала статей (по состоянию на 1 августа 2009 г.).

ТАБлицА 7. Наиболее популярные статьи портала Math-Net.Ru

\begin{tabular}{|c|c|c|}
\hline 1. & $\begin{array}{l}\text { О порядке приближения функций многих переменных линейными } \\
\text { положительными операторами конечного ранга } \\
\text { Р. К. Васильев } \\
\text { Математические заметки, 1993, 53:1, 3-15 }\end{array}$ & 1526 \\
\hline 2. & $\begin{array}{l}\text { Недооцененный Пуанкаре } \\
\text { В. И. Арнольд } \\
\text { Успехи математических наук, 2006, 61:1(367), 3-24 }\end{array}$ & 1270 \\
\hline 3. & $\begin{array}{l}\text { Разностный метод численного расчета разрывных решений уравнений } \\
\text { гидродинамики } \\
\text { С. К. Годунов } \\
\text { Математический сборник, } 1959, \mathbf{4 7}(89): 3,271-306\end{array}$ & 888 \\
\hline 4. & $\begin{array}{l}\text { Три подхода к определению понятия "количество информации" } \\
\text { А. Н. Колмогоров } \\
\text { Проблемъ передачи информачии, 1965, 1:1, 3-11 }\end{array}$ & 789 \\
\hline 5. & $\begin{array}{l}\text { Лингвистика по А. Т. Фоменко } \\
\text { А. А. Зализняк } \\
\text { Успехи математических наук, 2000, 55:2(332), 162-188 }\end{array}$ & 660 \\
\hline 6. & $\begin{array}{l}\text { Понятие энтропии в теории вероятностей } \\
\text { А. Я. Хинчин } \\
\text { Успехи математических наук, 1953, 8:3(55), 3-20 }\end{array}$ & 606 \\
\hline 7. & $\begin{array}{l}\text { Сергей Михайлович Никольский (к девяностолетию со дня рождения) } \\
\text { О. В. Бесов, С. В. Бочкарев, В. С. Владимиров, Е. А. Волков, } \\
\text { В. К. Дзядык, В. А. Ильин, Б. С. Кашин, Н. П. Корнейчук, } \\
\text { Л. Д. Кудрявцев, О. А. Олейник, Ю. С. Осипов, С. И. Похожаев, } \\
\text { С. Б. Стечкин, С. А. Теляковский, В. Н. Темляков, П. Л. Ульянов } \\
\text { Успехи математических наук, 1995, 50:6(306), 223-228 }\end{array}$ & 599 \\
\hline 8. & $\begin{array}{l}\text { Метод оптимальной фильтрации Калмана-Бьюси и его обобщения } \\
\text { М. Б. Прохоров, В. К. Саульев } \\
\text { Итоги науки и техники. Сер. Математический анализ, } 1977, \mathbf{1 4}, 167-207\end{array}$ & 583 \\
\hline 9. & $\begin{array}{l}\text { Интегральные и локальные характеристики сверхзвукового пульсирующего } \\
\text { детонационного прямоточного двигателя } \\
\text { В. Г. Александров, А. Н. Крайко, К. С. Реент } \\
\text { Математическое моделирование, 2003, 15:6, 17-26 }\end{array}$ & 574 \\
\hline 10. & $\begin{array}{l}\text { Дополнительные главы курса "Уравнения математической физики" } \\
\text { В. П. Михайлов, А. К. Гущин } \\
\text { Лекицонные курсы НОЦ, 2007, 7, 3-144 }\end{array}$ & 569 \\
\hline
\end{tabular}

Число в правом столбце табл. 7 - это индекс популярности статьи - вычисляется простым сложением количества обращений посетителей портала Math-Net.Ru к материалам соответствующей статьи: к странице с аннотацией, к полному тексту статьи или первой странице (если доступ к полному тексту закрыт), к списку цитируемой литературы и т. д. Данные приведены за все время наблюдения (с 17 апреля 2008 г.). Также можно посмотреть наиболее популярные статьи за последнюю неделю и за последний месяц. 
Списки наиболее цитируемых статей, наиболее цитируемых авторов и наиболее популярных статей доступны как для портала в целом, так и для каждого журнала в отдельности.

Отметим также, что любая публикация, упоминающаяся на портале Math-Net.Ru, сопровождается ссылками на несколько информационных систем, в которых она также присутствует: на Math-Net.Ru, на три международные реферативные базы данных - MathSciNet Американского математического общества, Zentralblatt MATH Европейского математического общества и ADS NASA (Астрофизическая информационная система, финансируемая Национальным агентством по аэронавтике и исследованию космического пространства США) - и ссылками по DOI на сайт издателя соответствующей публикации. Это относится к статьям основной базы данных “Журналы", к элементам списков цитируемой и цитирующей литературы к этим статьям. А также к "Основным публикациям" и "Полным спискам публикаций" персоналий, представленных на портале Math-Net.Ru.

Что влияет на величину импакт-фактора?

1. Актуальность полученных в статье результатов, популярность соответствующей тематики.

2. Временной разрыв между русскоязычной и англоязычной версиями журналов. Зарубежные читатели просто не успевают сослаться на статью, опубликованную в англоязычной версии журнала с большим отставанием от оригинальной статьи.

3. Узконаправленность журнала, количество авторов и статей, публикуемых в журнале. Как правило, узкая направленность журнала и небольшое количество статей, публикуемых в течение года, приводят к увеличению импакт-фактора.

4. Изучив представленные на портале Math-Net.Ru списки статей, дающих вклад в ИФМ, нетрудно заметить, что бо́льшая часть импакт-фактора формируется за счет так называемого самоцитирования - когда автор сам ссылается на свои предыдущие работы, а также когда одни статьи ссылаются на другие внутри самого журнала. Поэтому журналы с достаточно ограниченным и постоянным кругом авторов имеют больший импакт-фактор.

5. Доступность журнала для читателей (в первую очередь через Интернет). Совершенно очевидно, что чем быстрее статья станет доступной для широкого круга читателей, тем быстрее результаты, изложенные в ней, смогут повлиять на исследования других авторов, и, как следствие, цитируемость этой статьи увеличится.

На портале Math-Net.Ru доступ к полным текстам статей практически всех изданий либо полностью свободный, либо открыт с той или иной временной задержкой. Большим (если не сказать великим) событием этого года стало разрешение, полученное нами от Академиздатцентра "Наука" РАН, открыть доступ к архивам издаваемых им журналов с задержкой в три года.

Хотя, конечно, именно открытость текущих выпусков журналов важна для оперативного доступа читателей к новым публикациям и, соответственно, для увеличения цитируемости статей, что, безусловно, влияет на величину двухлетних импактфакторов журналов. Мы не призываем открыть доступ ко всем российским журналам, понимая, что это немедленно скажется на подписке, в первую очередь, на англоязычные версии журналов. Необходимо организовать продуманную подписку на электронные версии журналов. Сейчас подписку осуществляет Научная электронная библиотека (http://elibrary.ru), однако на большинство математических журналов такая подписка пока отсутствует.

Существует так называемый Российский индекс научного цитирования (РИНЦ), публикуемый на сайте Научной электронной библиотеки. Нам этот индекс представляется малоинформативным. В нем учитываются только ссылки из журналов, представленных в их собственной базе данных. Приведем таблицу, сравнивающую ИФМ и РИНЦ 2008 года для ряда математических журналов: 
ТАБЛИЦА 8. Сравнение ИФМ и РИНЦ 2008 г.

\begin{tabular}{|c|c|c|c|c|c|c|c|c|c|c|}
\hline & aа & al & $\mathrm{dm}$ & $\mathrm{da}$ & znsl & ivm & izv & $\mathrm{mz}$ & $\mathrm{mt}$ & $\mathrm{msb}$ \\
\hline \hline ИФМ & 0.340 & 0.493 & 0.212 & 0.224 & 0.179 & 0.095 & 0.310 & 0.283 & 0.171 & 0.506 \\
\hline РИНЦ & 0.022 & - & 0.088 & 0.208 & 0.015 & - & 0.054 & 0.030 & 0.094 & 0.113 \\
\hline
\end{tabular}

\begin{tabular}{|c|c|c|c|c|c|c|c|c|c|c|}
\hline & $\mathrm{mm}$ & $\mathrm{mmj}$ & $\mathrm{ppi}$ & $\mathrm{sjim}$ & $\mathrm{smj}$ & $\mathrm{tmf}$ & $\mathrm{tm}$ & $\mathrm{umn}$ & $\mathrm{fpm}$ & faa \\
\hline \hline ИФМ & 0.123 & 0.444 & 0.354 & 0.181 & 0.528 & 0.735 & 0.363 & 0.488 & 0.207 & 0.513 \\
\hline РИНЦ & 0.193 & - & 0.356 & 0.161 & 0.108 & 0.107 & 0.098 & 0.103 & - & 0.051 \\
\hline
\end{tabular}

Еще раз хотим отметить следующее. Из всего, что касается цитирования, наиболее важной для нас является возможность видеть не только пристатейные списки цитируемой литературы, но и списки работ, цитирующих данную статью. Такая возможность позволяет, например, понять, как и кем полученные результаты использовались и развивались в дальнейших публикациях. Все остальное, в том числе импакт-факторы журналов, - "бесплатное" приложение.

В заключение несколько слов о перспективах развития системы Math-Net.Ru.

Конечно, в первую очередь мы будем стремиться вовлечь в нашу орбиту как можно больше новых журналов. В том числе в области прикладной математики, информатики, механики и физики.

Будет продолжаться обработка списков цитируемой литературы, а также поиск всевозможных ссылок на статьи, представленные в базе данных Math-Net.Ru.

Наряду с двухлетними ИФМ будем считать и пятилетние импакт-факторы. На них, в частности, не такое сильное влияние оказывает оперативность доступа к текущим выпускам журналов.

Мы предполагаем создать единую базу данных всех ссылок, собранных на портале Math-Net.Ru (списки цитируемой и цитирующей литературы, а также "основные публикации" на страницах персоналий). Уже сейчас на попадание в такую базу данных претендует более 600 тысяч записей. Здесь, помимо статей в российских и зарубежных журналах, будут присутствовать книги, труды конференций, диссертации, препринты и т. д. В частности, сюда попадут все те публикации, на которые хотя бы раз сослались авторы "матнетовских" статей.

Основная нетривиальная задача, которая должна быть решена при создании этой базы данных, - "сшивка" ссылок на одну и ту же публикацию. Проблема в том, что оформляются ссылки в различных источниках совершенно по-разному и, кроме того, имеется масса ошибок и опечаток при оформлении библиографических списков.

Итогом создания базы данных цитируемой и цитирующей литературы станет система поиска публикаций по различным параметрам. Результатом поиска будет не только искомая публикация, но и список цитируемых и цитирующих ее работ.

Общероссийский математический портал Math-Net.Ru создан и развивается Математическим институтом им. В.А. Стеклова РАН совместно с Отделением математических наук РАН в рамках программы фундаментальных исследований Отделения математических наук РАН "Алгебраические и комбинаторные методы математической кибернетики и информационные системы нового поколения", программ фундаментальных исследований Президиума РАН "Проблемы создания национальной научной распределенной информационно-вычислительной среды на основе развития GRID технологий и современных телекоммуникационных сетей" и "Фундаментальные проблемы системного программирования", а также целевой программы Президиума РАН "Информатизация". 


\section{Список литературы}

[1] А. Б. Жижченко, А. Д. Изаак, "Информационная система Math-Net.Ru. Применение современных технологий в научной работе математика", УМH, 62:5 (2007), 107-132; англ. пер.: А.В. Zhizhchenko, A.D. Izaak, "The information system Math-Net.Ru. Application of contemporary technologies in the scientific work of mathematicians", Russian Math. Surveys, 62:5 (2007), 943-966.

[2] R. Adler, J. Ewing, P. Taylor, Citation Statistics, A report from the International Mathematical Union (IMU) in cooperation with the International Council of Industrial and Applied Mathematics (ICIAM) and the Institute of Mathematical Statistics (IMS) http://www . mathunion.org/fileadmin/IMU/Report/CitationStatistics.pdf, 2008.

[3] С. М. Гусейн-Заде, "Вокруг импакт фактора", УМH, 59:5 (2004), 186-188; англ. пер.: S. M. Gusein-Zade, "About the impact factor", Russian Math. Surveys, 59:5 (2004), 1005-1007.

[4] С. С. Кутателадзе, "Игра в цифирный бисер", Вестн. Владикавказ. науч. центра, 9:2 (2009), 52-53; S. S. Kutateladze, The game of cipher beads, arXiv:0905.3330.

[5] Т.А. Полилова, Инфраструктура научных публикаиий, Препринты ИПМ им. М. В. Келдыша, № 15, 2009, 30 c.; http://library.keldysh.ru/preprint.asp ?id=2009- 15 .

[6] В. В. Писляков, “Зачем создавать национальные индексы цитирования?", Науч. u mеx. б-кu, 2007, № 2, 65-71.

[7] В. В. Писляков, Е. Л. Дьяченко, “Эффект Матфея в цитировании статей российских ученых, опубликованных за рубежом", Научно-техническая информация. Сер. 2. Информачионные прочессы и системы, 2009, № 3, 19-24; V. V. Pislyakov, E. L. Dyachenko, "The Matthew Effect in citing the papers of Russian scientists published abroad", Automat. Document. and Math. Linguistics, 43:2 (2009), 76-82. 